\title{
The Exploration of the Transneptunian Population
}

\author{
Brian G. Marsden \\ Harvard-Smithsonian Center for Astrophysics, Cambridge, MA 02138
}

\begin{abstract}
The recognition of the extensive population of bodies in the outer Solar System has been arguably the most significant discovery about the Solar System from groundbased observations during the twentieth century. Our understanding of the transneptunian population is still quite flawed in its details, although a plausible picture has been emerging.
\end{abstract}

\section{Introduction}

Now that a body of the evident dimensions and mass of Pluto has been revealed, is there any reason to suppose that there are not other, probably similarly constituted, members revolving around the Sun outside the orbit of Neptune? Indeed, it may ultimately be found that the Solar System consists of a number of zones, or families, of planets, one within the other. As a matter of fact, astronomers have recognized for more than a century that this system is composed successively of the families of the terrestrial planets, the minor planets, and the giant planets. Is it not likely that in Pluto there has come to light the first of a series of ultra-Neptunian bodies, the remaining members of which still await discovery but which are destined eventually to be detected?

The above statement by Leonard (1930) is a quite remarkable description of the Solar System, given that it was written only months after the discovery of Pluto. Like others, he had to contend with the idea that Pluto was comparable in size to the terrestrial planets, but in considering Pluto as one of many members in a new "zone" he was way ahead of subsequent commentators. Furthermore, as time went by, it became clear that there are no other transneptunian members at least consistently comparable to Pluto in apparent brightness, leading to another prophetic remark (except that it did not envisage the development of CCD technology!) by Whipple (1964):

If the largest comets in the postulated belt have radii not much exceeding $100 \mathrm{~km}$, or approximately $1 / 30$ Pluto's diameter, their apparent magnitude would be approximately 22 and the chance of discovery remote, even though a number of such comets might exist. 


\section{2. $\quad 1992-1995$}

The story of the early discoveries, beginning in 1992, of these postulated smaller members of the transneptunian belt is now well known, the situations as of mid-1993 and mid-1994 having been reviewed, for example, by Luu (1994) and by Marsden (1996), respectively. Although the first two cases, where the initial computation of direct, circular orbits placing these objects 11-12 AU beyond the orbit of Neptune was later demonstrated to be closely representing the truth, the next four cases involved such solutions that required passage only 2-5 AU from Neptune. The hypothesis that these four objects did not in fact currently approach Neptune anything like as closely and were instead near the perihelia of somewhat eccentric orbits, like that of Pluto in 2:3 mean-motion resonance with Neptune, was subsequently shown to be correct for three of them. Five of the six transneptunian objects (TNOs) found in 1992 and 1993 therefore have orbits that are quite stable over a considerable fraction of the lifetime of the Solar System. The sixth object, 1993 RP, has not been observed since two nights after its discovery.

Four of the 12 transneptunian candidates discovered in 1994 are lost, as are as many as eight of the 17 discovered in 1995. Since most of the lost objects did not go unsought, it was always rather evident that this failure to recover was largely because the lost objects had orbital eccentricities significantly larger than had been assumed on the basis of observations generally extending no longer than a month at the discovery opposition. However, more complete recovery attempts with the required large-aperture, small-field telescopes would put a significant strain on resources that might more profitably be used to make new discoveries. Many objects could be recognized as cubewanos or plutinos, i.e., the two categories already noted from the 1992-1993 discoveries, and together with a handful of other resonant objects of low to moderate orbital eccentricity, they were considered sufficiently representative of the population.

\section{Centaurs and scattered-disk objects}

That there are beyond Neptune at any time physically comparable objects of low orbital eccentricity and near the aphelia of high-eccentricity orbits of lowto-moderate inclination was clear from study of the centaurs, three of which, with their perihelia in the vicinity of Saturn, were discovered between 1977 and 1993. Such orbits are clearly rather unstable on a timescale as short as $10^{4}$ years (Kowal, Liller, \& Marsden 1979), and a transneptunian population of cubewanos and plutinos, even if dynamically stable for, say, $10^{8}$ years, does therefore provide a likely source for the centaurs. Given that subsequent dynamical evolution of the centaurs can clearly produce orbits of the type occupied by Jupiter-family comets, and that the first centaur discovered, $(2060)=95 \mathrm{P} /$ Chiron, does show cometary activity, there is also an obvious physical sink for the centaurs.

But a low-eccentricity, low-inclination, transneptunian population can obviously also evolve into a high-eccentricity, low-to-moderate-inclination population of larger orbits, and in the absence of further external perturbers or physical dissolution or disruption, there would be a tendency for the perihelia to remain in the general vicinity of the orbit of Neptune. If centaurs exist, so should objects 
of this "scattered-disk" variety-all the more so, since they are less likely to be further disturbed. The first such object to be recognized was $1996 \mathrm{TL}_{66}$ (Luu et al. 1997). It was the availability of observations in a third month at the discovery opposition that showed this object not to be a plutino, but to have an orbit of eccentricity 0.6. This first scattered-disk object, or SDO, was also mentioned by Marsden (1999) in a review that discussed approaches to the giant planets, specifically showing that cubewanos and plutinos make minimum approaches to Neptune of 9 and $11 \mathrm{AU}$, respectively (with plutinos approaching Uranus to 7 $\mathrm{AU}$ ), within several millennia of the present time, while $1996 \mathrm{TL}_{66}$ passes some 6 AU from Neptune.

Recognition of the second SDO, $1998 \mathrm{XY}_{95}$, was brought about only because of the persistence of an amateur astronomer in following this object up after its discovery month (Marsden \& Offutt 2000). The year 1999 alone saw a doubling in the number of known TNOs, 10 percent of them as faint as magnitude 25.5-26.5. Ten of the 1999 discoveries that could be recovered at their 2000 oppositions were then established to be SDOs, a point that confirms the likelihood that several of the TNOs lost in the early years were also in this class.

If a distinction between centaur and SDO indeed needs to be made, it is unclear whether this should be on the basis of orbital mean distance or perihelion distance. While it may be tempting to make the distinction at mean distance $30 \mathrm{AU}$ (i.e., Neptune's mean distance), the rather steady progression of values through that distance does not suggest any physical significance. On the other hand, the perihelion-distance distribution shows a convenient gap between 22 and $30 \mathrm{AU}$, with then a steady progression of SDO values extending to a 38-AU maximum. Whether or not the point has any significance (e.g., as regards the presence of a more massive body in a regular orbit), it can be noted that the largest SDO aphelion distances show a concentration around $200 \mathrm{AU}$. This is also the aphelion distance of $1999 \mathrm{TD}_{10}$, an object with perihelion distance 12 $\mathrm{AU}$ that is perhaps more readily classified as a centaur.

\section{1:2 Neptune resonance and beyond}

Given the existence of plutinos at mean distance 39-40 AU, there has for some time been speculation as to whether there are any objects in 1:2 libration with Neptune that can also avoid encounters with that planet. The relative number of 2:3 and 1:2 librators is a matter of some significance, because it allows one to distinguish among hypotheses for the origin of the transneptunian system (Malhotra 1995). With their mean distances of 47-48 AU (i.e., just beyond the cubewanos) and their perihelia near the orbit of Neptune, the 1:2 librators have orbital eccentricities approaching 0.4 . Two reasonably definite cases, 1997 $\mathrm{SZ}_{10}$ and $1996 \mathrm{TR}_{66}$ (Marsden, Levison, \& Malhotra 1998) have been recognized (but are very much in need of further observation), with a possible third twoopposition object and four single-opposition "guesses".

Whether there exist TNOs in regular (i.e., non-SDO) orbits beyond the 1:2 resonance is unclear. Certainly, objects have been observed beyond a heliocentric distance of $50 \mathrm{AU}$, but there is no indication they are part of a different population. The most distant object so far observed, at a heliocentric distance 
of around $60 \mathrm{AU}$, is $1999 \mathrm{DG}_{8}$. It was recorded on only two consecutive nights, however, and could easily be an SDO.

\section{Concluding remarks}

Of the 133 multiple-opposition TNO-centaur population, the cubewanos dominate at $52 \%$, with plutinos at $22 \%$, followed by centaurs and SDOs combined at $19 \%$. The remainder includes the 1:2 librators, $3: 5$ librators and the single $3: 4$ librator. Between the 3:4 and 2:3 regions, at heliocentric distance $38 \mathrm{AU}$, there appear also to be two objects in nearly circular orbits of long-term stability (Duncan, Levison, \& Budd 1995); their orbital inclinations are $5^{\circ}$ and $25^{\circ}$. Generally, the resonant TNOs have small to modest inclinations, ranging up to $20^{\circ}$. The inclination distribution for the cubewanos is intriguing, with some $60 \%$ under $5^{\circ}$, with half of the remainder extending to about $17^{\circ}$ and the other half still uniformly from $20^{\circ}$ to $32^{\circ}$. These high-inclination cubewanos almost seem to form a separate population: they also generally have noticeably smaller perihelion distances than the low-inclination cubewanos, although the orbital orientations still keep the objects far from Neptune.

To specify a more meaningful distribution of the TNOs one should consider only the intrinsically brighter objects. From those brighter than absolute magnitude 7.5 it follows that $66 \%$ are cubewanos and $12 \%$ are plutinos. The $9 \%$ SDO contribution is probably still an underestimate, while centaurs and 1:2 librators each account for $5 \%$. Each remaining $1 \%$ then applies to the 3:5 librators, the $3: 4$ librators and the "38-AU" group.

\section{References}

Duncan, M. J., Levison, H. F., \& Budd, S. M. 1995, AJ, 110, 3073

Kowal, C. T., Liller, W., \& Marsden, B. G. 1979, in IAU Symp. 81, Dynamics of the Solar System, ed. R. L. Duncombe (Dordrecht: Reidel), 245

Leonard, F. C. 1930, ASP Leaflet, 30

Luu, J. X. 1994, in IAU Symp. 160, Asteroids, Comets, Meteors 1993, ed. A. Milani, M. Di Martino, \& A. Cellino (Dordrecht: Kluwer), 31

Luu, J. X., Marsden, B. G., Jewitt, D., Trujillo, C. A., Hergenrother, C. W., Chen, J., \& Offutt, W. B. 1997, Nature, 387, 573

Malhotra, R. 1995, AJ, 110, 420

Marsden, B. G. 1996, in ASP Conf. Ser. Vol. 107, Completing the Inventory of the Solar System, ed. T. W. Rettig \& J. M. Hahn (San Francisco: ASP), 193

Marsden, B. G. 1999, Celest. Mech., 73, 51

Marsden, B. G., Levison, H. F., \& Malhotra, R. 1998, IAU Circ., 7073

Marsden, B. G. \& Offutt, W. B. 2000, BAAS, 32, 689

Whipple, F. L. 1964, Proc. Nat. Acad. Sci., 51, 711 